\title{
Conflicto social en Chile: minería de opinión en Twitter durante la primera semana de la protesta chilena del año 2019
}

Social Conflict in Chile: mining opinion on Twitter during the first week of the Chilean protest in 2019

Conflito social no Chile: mineração de opinião no Twitter durante a primeira semana do protesto chileno de 2019

Ricardo RIVERA GALLARDO

Universidad de Santiago de Chile (Chile)

rarivera2@uc.cl

Chasqui. Revista Latinoamericana de Comunicación

N. ${ }^{\circ}$ 148, diciembre 2021 - marzo 2022 (Sección Diálogo de saberes, pp. 219-238)

ISSN 1390-1079 / e-ISSN 1390-924X

Ecuador: CIESPAL

Recibido: 28-07-2O21 / Aprobado: 12-11-2021 


\title{
Resumen
}

Esta investigación analizó los procesos de canalización del malestar en Twitter, durante el estallido social chileno del año 2019. En primer lugar, se realizó una revisión de la literatura concerniente a las últimas protestas y movimientos sociales chilenos. En segundo lugar, se analizaron las investigaciones relacionadas a la minería de opinión en Twitter. En tercer lugar, se capturaron los comentarios de los usuarios entre los días 18 y 26 de octubre (el momento más espontaneo del estallido), a través de técnicas de minería de opinión, dichos comentarios fueron almacenados y analizados para identificar y reconocer los posts que estaban relacionados a los hashtags de los trending topic en Chile. Por último, se procedió al análisis los datos para verificar el rol de la figura del presidente y las otras palabras presentes, con estos datos se puede señalar que existe una re-significación del malestar por medio de la figura de Sebastián Piñera, su gobierno y las instituciones relacionadas a este, los cuales se transforman en objetos de discurso y de representación de lo antagónico a la idea de pueblo.

Palabras clave: redes sociales, significante vacío, estallido social, Twitter y minería de opinión.

\begin{abstract}
This research analyzed the processes that channeled the unrest on Twitter during the Chilean social outbreak in 2019. First, a review of the literature concerning the latest Chilean protests and social movements was conducted. Second, investigations related to opinion mining on Twitter were analyzed. Third, user comments were collected between October 18 and 26 (the most spontaneous moment of the outbreak), through opinion mining techniques; these comments were stored and analyzed to identify and recognize the posts that were related to the hashtags of the trending topics in Chile. Finally, this data was analyzed to verify the role of the figure of the president together with the other words found. With these data it can be stated that there is a re-significance of the malaise through the figure of Sebastián Piñera, his government and the institutions related to him. These are transformed into objects of discourse and representation of what is antagonistic to the idea of the people.
\end{abstract}

Keywords: social media, empty signifier, social pop, Twitter, and opinion mining.

\section{Resumo}

Esta pesquisa analisou os processos de canalização do mal estar no Twitter, durante a explosão social chileno de 2019. Primeiramente, foi realizada uma revisão de literatura sobre os últimos protestos e movimentos sociais chilenos. Em segundo lugar, foram analisadas as pesquisas relacionadas à mineração de opinião no Twitter. Em terceiro lugar, foram capturados os comentários dos usuários entre 18 el 26 de outubro (o momento mais espontâneo da explosão 
social), por meio de técnicas de mineração de opinião. Esses comentários foram armazenados e analisados para identificar e reconhecer as postagens que estavam relacionadas às hashtags dos trending topics no Chile. Por último, os dados foram analisados para verificar o papel da figura do presidente e as demais palavras presentes. Com esses dados, pode-se apontar que há uma ressignificação do mal estar mediante a figura de Sebastián Piñera, seu governo e as instituições relacionadas, que se transformam em objetos de discurso e representação do que é antagônico à ideia de povo.

Palavras-chave: redes sociais, significante vazio, explosão social, Twitter e mineração de opinião

\section{Introducción}

Esta investigación parte de la complejidad social del discurso que se evidenció en la primera semana de las protestas chilenas. El peso de algunas las palabras claves contiene una relevancia especial en el escenario político e ideológico del país, ya que a partir de las palabras se logra determinar los límites de lo aceptable (Foucault, 1966). Sin embargo, las preguntas tienen relación con una reflexión aún más compleja, puesto que las palabras tienden a transformarse de forma paulatina en el tiempo, a pesar de eso, las imágenes que están asociadas a esa palabra o el significado unido a ellas, sufren cambios abruptos según los contextos (Le Bon, 1895). En términos de Ernesto Laclau y Chantal Mouffe (1985) la sociedad y sus demandas se constituyen en torno a los límites antagónicos de esta, desde donde todos los objetos, en tanto significados (o sea las formas discursivas designadas), se constituyen como objetos de discurso para la sociedad, por medio de una designación (o sea un significante). Acá es donde aparece la conceptualización de Laclau (2005) sobre "significante vacío", con el cual, señalan ellos, buscan explicar el fenómeno de la canalización del malestar social en un objeto discursivo, ya sea una figura pública, una institución o un concepto abstracto.

En específico, el "significante vacío" es una equivalencia simbólica entre una concatenación de demandas sociales y un objeto discursivo designado. Por ende, el "significante vacío" nace de la imposibilidad de representar lo real en el orden simbólico, y a su vez, de la posibilidad de representar una heterogeneidad de significados que varían en tiempo. El "significante vacío" se encuentra en un estado de latencia en el escenario político-social, como un "elemento" abierto a la posibilidad y al cambio de las coordenadas de su significación. Para ambos autores, este "elemento" es un estado previo a la manifestación del fenómeno de canalización discursiva del malestar social, ya que cuando se manifiesta este elemento en la sociedad se expresa como un "momento populista" (Laclau, 
2005), donde el "significante vacío" logra expresar una equivalencia con una diversidad de demandas sociales. Esta instancia logra resignificar el escenario político-social. Sin embargo, el "momento populista" está abierto a una nueva articulación de demandas sociales y por esta razón a una nueva re-significación, porque el "significante vacío" mantiene siempre la posibilidad de una nueva concatenación de equivalencias nueva.

En el caso del estallido social chileno, se utilizó el método de análisis propuesto por Ernesto Laclau (2005), ya que en este fenómeno social se puede asociar a un momento populista. La existencia de una multiplicidad de demandas diversas de la sociedad chilena, pero que se articularon en “significantes vacíos". El presidente Sebastián Piñera, el gobierno, carabineros y otras figuras e instituciones se hicieron presentes en los posts de Twitter. Ante esto surgen las siguientes preguntas: en primer lugar, ¿existe una relación de la figura del presidente Sebastián Piñera y las distintas demandas surgidas en Twitter durante los primeros días de las protestas iniciadas el 18 de octubre? En segundo lugar, ¿existieron más actores e instituciones que respondieran a la idea de un "significante vacío" para los usuarios de Twitter durante esa primera semana de manifestaciones? En tercer lugar, ¿podemos hablar que existió un momento populista en el estallido social chileno en base a los datos capturados desde Twitter? En cuarto lugar, ¿se cumplen los parámetros señalados por Ernesto Laclau y Chantal Mouffe en el análisis de Twitter? Por último, ¿la minería de datos puede servir para este tipo de análisis?

Las manifestaciones del 18 de octubre tienen como hito de inicio las protestas de los estudiantes secundarios por el alza del pasaje de adulto en el metro de Santiago (Mayol, 2019; Ruiz, 2020; Alé, Duarte y Miranda, 2021). Una demanda que escapaba de la influencia directa en la vida de los estudiantes, sin embargo, existía una relación con la situación de sus familias, la conexión se daba en un segundo nivel, porque eran sus padres los más afectados con el alza del pasaje. Por este motivo, la protesta rápidamente se abrió paso hacia un grupo más heterogéneo de la sociedad y hacia una sería de demandas más amplias que el alza del pasaje de metro (Alé, Duarte y Miranda, 2021). Las manifestaciones previas al 18 de octubre se conectaron con el malestar de una parte importante de la ciudadanía, mientras tanto los distintos poderes del Estado buscan responder a la protesta de los estudiantes diciendo que simplemente era un problema delictual (Mayol, 2019). Por otro lado, previo al estallido de octubre, estaban los múltiples movimientos sociales que eran una nueva forma de organización (Garcés, 2011). En este sentid, todas las demandas por separado se expresaron en distintos momentos de la historia reciente (Alé, Duarte y Miranda, 2021), pero el 18 de octubre unió a todas estas demandas, permitiendo que se canalizara en algunos "significantes vacíos". Esto se puede constatar con la información que se plasmó en redes sociales con las palabras claves que más se repitieron. 
La hipótesis central de la investigación es que el 18 octubre fue un momento de re-figuración de "significantes vacíos". En primer lugar, hay que señalar que el presidente Sebastián Piñera, como figura máxima de gobierno y centro de la discusión política, se cristalizó como una figura antagónica, el estallido relacionado con dos décadas de movilización social, permitió unificar una gran cantidad las demandas que habían sido frustradas anteriormente, esto derivó en la conformación de otro en oposición al antagonista. Esto queda reflejado en la frecuencia en la que aparece el nombre del presidente en Twitter y el rol que jugará su figura en los primeros días de la movilización. Tal como se mencionó, el fenómeno del objeto de discurso, Sebastián Piñera, depende del momento populista y la configuración de un significante vacío que cuestione su poder. La unidad de una serie de demandas es necesario para constituir una "significante vacío", en el caso del estallido social las demandas de transporte, en primera instancia, conectaron con las de educación, vivienda, trabajo, género, movimientos por la diversidad, ecológicos, salud, etc. Esto último queda reflejado en una serie de significantes que constituyen la idea pueblo, acá podemos identificar sujetos, acciones y adjetivos, los cuales están relacionados directamente con el momento populista planteado por Ernesto Laclau y Chantal Mouffe. Por último, señalar que la minería de opinión a través del procesamiento natural permitió analizar el fenómeno del significante vacío en los momentos de tensión social y articulación de demandas.

En síntesis, el objetivo general de la investigación fue estudiar la relación entre la frecuencia de las palabras y la constitución de los significantes vacíos, principalmente la constitución de pueblo y su versión antagónica representada por el presidente Sebastián Piñera. En esta misma dirección, el segundo objetivo tiene relación con identificar las palabras que conectaron con los actores e instituciones que también se configuraron como "significantes vacíos" para los usuarios de Twitter durante esa primera semana de manifestaciones. El tercer objetivo, propuesta en esta investigación, es dilucidar si existió un momento populista en el estallido social chileno en base a los datos capturados desde Twitter. El cuarto objetivo, es definir si se cumplen los parámetros señalados por Ernesto Laclau y Chantal Mouffe en el análisis de Twitter. Por último, definir si la minería de datos sirve para este tipo de análisis.

\section{Marco teórico}

Los fenómenos de interés para las ciencias son hechos complejos que pueden ser analizados y estudiados desde una gran cantidad de premisas y métodos (Kuhn, 1962; Lakatos, 1983). Esto se ve todavía más claro cuando estudiamos a la sociedad y su relación con las nuevas tecnologías (Vinck, 2018). En el marco de esta premisa, es que este estudio pretende analizar las palabras claves en el proceso de significación de un malestar social, en particular, el estallido social ocurrido en Chile el año 2019. Sin embargo, esta investigación tomó como 
referencia la primera semana de manifestación, cuando las protestas tomaron por sorpresa el espacio de la política chilena (Akram, 2020; Contardo, 2020; Mayol, 2019; Ruiz, 2020; Alé, Duarte y Miranda, 2021; Cuevas y Sáez, 2021).

Esta investigación tomó como referencias los estudios sociológicos de los movimientos sociales, los estudios políticos del discurso y los estudios sobre el procesamiento de opiniones en Twitter a través del Procesamiento de Lenguaje Natural, ya que, a través de estos, se puede ordenar y caracterizar una gran cantidad de información. En sentido, administrar una multiplicidad de temáticas relacionadas a las protestas de las últimas dos décadas en Chile, que además comparten un contexto y relaciones difusas, obliga a desplegar un abanico de visiones y estudios sobre las protestas sociales y los movimientos del último tiempo. Por esta razón, para realizar el análisis también se consideró las investigaciones sobre los nuevos movimientos sociales y sus características, específicamente con las líneas relacionadas a los nuevos movimientos sociales propuestas por Alain Touraine $(1993,2006)$ y a la influencia de los nuevos medios de comunicación, como señalan Tilly y Wood (2010), Castells (2000) y Bennett (2003).

Con respecto a las articulaciones discursivas que ocurrieron en la primera semana de los acontecimientos de octubre del 2019, se definió estudiar el fenómeno desde la reoría populista. El "populismo” según Laclau (1971) corresponde a una dimensión de las democracias liberales, en las cuales surge un antagonismo, libre de las premisas economicistas, entre la tensión social generada por las condiciones propias de las relaciones en el capitalismo y las demandas frustradas del pueblo. Este último concepto, aparece para aglutinar a la amplia gama de sectores de la sociedad que están condicionados por el sistema capitalista, pero que se articulan discursivamente fuera de los parámetros del sistema productivo, estas demandas siempre surgirán desde las asimetrías entre la plenitud de la comunidad y el particularismo de los sitios de enunciación (Laclau, 2008). En este sentido, para Laclau (2005) la frustración de las demandas sociales en el marco del sistema capitalista y de la democracia liberal, origina un proceso de equivalencia entre las demandas, lo cual permite la unión y la generación de un "significante vacío". Este momento equivalencia de demandas heterogéneas permite el surgimiento del pueblo y de las fronteras políticas (Laclau, 2005), estas no necesariamente son lógicas o que dependan estrictamente de las condiciones de producción del sistema capitalista (Laclau, 1971).

Por último, nosotros tomamos como referencia algunas investigaciones sobre procesamiento de leguaje natural, principalmente enfocados en los procesos de captura de los posts en Twitter en situaciones de crisis. El informe desarrollado por Marcelo Mendoza, Bárbara Poblete y Carlos Castillo (2010) con respecto a las reacciones en Twitter después del terremoto del año 2010 en Chile, permitió identificar una serie de características que se desenvuelven en un momento de crisis en esta red social. A través de los hashtags se relacionaron 
los posts que mencionaban las temáticas que les permitió describir el proceso del terremoto. Luego, con el análisis de las redes y las palabras claves concluyeron que en una crisis existe un bajo nivel de entropía con respecto a las palabras que aparecen en las redes, esto quiere decir que es más fácil visibilizar las palabras e ideas relacionadas en este tipo de contextos, incluso, el bajo nivel de entropía en una crisis propicia que la mayoría de los comentarios en la red social estén relacionados a la contingencia, por lo cual, la captura de los post en Twitter recoge una gran cantidad de opiniones en estado natural.

\section{Metodología}

Como se mencionó en los apartados anteriores, el interés de esta investigación está puesto en las palabras claves, presentes en la minería de texto realizada en Twitter. La hipótesis que se presentó forma parte del análisis de estudio de la teoría de "populismo" de Ernesto Laclau y Chantal Mouffe (1985), ya que se definió que existe una relación de la figura del presidente Sebastián Piñera y las distintas demandas surgidas en Twitter; en segundo lugar, se buscaron otros "significantes vacíos"; en tercer lugar, se identificaron los parámetros para definir la existencia de un momento populista en el estallido social chileno, en base a los datos capturados; en cuarto lugar, se buscó analizar si se cumplían los parámetros señalados por Ernesto Laclau y Chantal Mouffe en el análisis de Twitter; por último, se propuso definir si la minería de opinión es útil para este tipo de análisis.

Lo primero que se realizó fue una investigación sobre las reflexiones sociológicasy políticas del estallido socialy los movimientos sociales relacionados de las últimas dos décadas, por eso, se tomó como referencias las principales publicaciones sobre los movimientos sociales en Chile y las reflexiones políticas sobre el estallido social; esto permitió identificar rápidamente las palabras y conceptos más relacionados al fenómeno del estallido. Lo segundo fue identificar la red social más próxima a las necesidades de la investigación, en este caso fue Twitter dado la facilidad para capturar los datos y por ser la red social más relacionada a la política. En tercer lugar, se generó un corpus lingüístico con 9.163.437 (este número es considerante los retweet) de comentarios escritos por los usuarios de Twitter, esto se consiguió por medio de la plataforma APIs ${ }^{1}$ que permitió obtener gran cantidad de información en tiempo real, sin embargo, se necesitó complementar con Logstash ${ }^{2}$ (software desarrollado por elastic. co) para administración de los mensajes, después se programó una lista de keywords principales, permitiendo indicar a Twitter que solo se debían obtener comentarios que contengan una o varias de las palabras claves.

En quinto lugar, se proceso la información, todos los comentarios, luego, se analizaron y se limpiaron a través de Python y la librería Spark2 (pyspark).

1 Interfaz de programación de aplicaciones.

2 https://www.elastic.co/es/products/logstash 
Los pasos de este proceso fueron los siguiente: se tomaron solo los campos de ID (usuario, comentarios y hashtags); se identificaron y eliminaron todos los comentarios catalogados como "referenciados"; y se limpió gran parte de twitts que no tenían relación con los acontecimientos relacionados a las actividades en chile. En sexto lugar, una vez que se obtuvo conjunto de comentarios completos y legibles, estos fueron cargados a GCP (Google Cloud Platform), específicamente a la herramienta llamada BigQuery, esto permitió ya desde ese momento tener datos legibles y disponibles a través del lenguaje de consultas SQL. Por último, se utilizó el software de visualización de Tableau para graficar las palabras.

Tabla resumen con universo de la información recopilada: Tokens, fecha y frecuencias absolutas de cada concepto (Figura 1)

\begin{tabular}{|c|c|c|c|c|c|c|c|c|c|c|}
\hline \multirow{2}{*}{$\begin{array}{l}\text { Suma de frecuencia } \\
\text { Etiquetas de fila }\end{array}$} & Etiquetas de columna $\nabla$ & \multirow[b]{2}{*}{ 20-oct } & \multirow[b]{2}{*}{ 21-oct } & \multirow[b]{2}{*}{ 22-oct } & \multirow[b]{2}{*}{ 23-oct } & \multirow[b]{2}{*}{ 24-oct } & \multirow[b]{2}{*}{ 25-oct } & \multirow[b]{2}{*}{ 26-oct } & \multirow{2}{*}{\multicolumn{2}{|c|}{ 27-oct Total general }} \\
\hline & 19-oct & & & & & & & & & \\
\hline presidente & 43.652 & 49.067 & 46.618 & 33.285 & 31.746 & 32.570 & 21.686 & 26.804 & 13.534 & 298.962 \\
\hline renunciapiñera & 5.225 & 19.659 & 26.220 & 47.780 & 30.938 & 10.290 & 6.245 & 8.596 & 1.004 & 155.957 \\
\hline piñera & 23.155 & 20.192 & 24.356 & 17.304 & 12.307 & 8.916 & 6.996 & 13.469 & 2.214 & 128.909 \\
\hline metro & 26.727 & 16.149 & 15.227 & 12.682 & 14.022 & 14.818 & 11.051 & 9.431 & 2.992 & 123.099 \\
\hline plazaitalia & 10.549 & 8.730 & 8.051 & 6.926 & 6.326 & 8.117 & 6.666 & 7.304 & 1.868 & 64.537 \\
\hline noticia & 6.778 & 6.550 & 9.024 & 8.380 & 8.226 & 9.418 & 5.691 & 5.552 & 1.691 & 61.310 \\
\hline chile & 8.426 & 8.864 & 9.443 & 9.777 & 7.573 & 5.816 & 4.031 & 5.620 & 1.039 & 60.589 \\
\hline pacos & 12.498 & 12.831 & 9.964 & 6.918 & 5.110 & 4.697 & 2.425 & 2.644 & 1.207 & 58.294 \\
\hline chiledesperto & 8.559 & 7.973 & 10.561 & 6.424 & 3.117 & 3.057 & 3.075 & 5.328 & 753 & 48.847 \\
\hline estopasaenchile & & & 15.921 & 22.337 & 4.497 & 1.647 & & & & 44.402 \\
\hline milicos & 6.530 & 6.165 & 5.309 & 14.378 & 5.078 & 3.756 & 1.533 & 1.286 & & 44.035 \\
\hline toquedequeda & 6.652 & 16.297 & 4.467 & 3.377 & 2.331 & 1.591 & 919 & 1.622 & & 37.256 \\
\hline piñeradictador & & & 8.443 & 14.949 & 5.445 & 2.527 & 1.453 & 2.093 & 1.883 & 36.793 \\
\hline sebastianpinera & 5.283 & 4.257 & 4.763 & 5.737 & 4.469 & 3.352 & 2.988 & 4.989 & 649 & 36.487 \\
\hline gente & 5.479 & 5.441 & 4.750 & 4.904 & 4.235 & 3.865 & 2.661 & 2.743 & 924 & 35.002 \\
\hline culiao & 7.674 & 6.436 & 5.082 & 4.539 & 2.847 & 2.181 & 1.081 & 2.222 & 425 & 32.487 \\
\hline biobio & & & 2.760 & 8.193 & 6.680 & 5.277 & 3.281 & 3.689 & 556 & 30.436 \\
\hline pais & 3.838 & 4.096 & 3.757 & 3.881 & 3.323 & 3.289 & 1.891 & 2.356 & 956 & 27.387 \\
\hline pueblo & 3.459 & 4.139 & 4.932 & 4.344 & 2.949 & 2.183 & 1.539 & 2.354 & 478 & 26.377 \\
\hline hueon & 4.492 & 4.222 & 3.373 & 3.966 & 2.732 & 2.374 & 1.152 & 2.048 & & 24.359 \\
\hline ministro & & 2.741 & 3.376 & 3.026 & 2.716 & 6.463 & 2.287 & 3.012 & 541 & 24.162 \\
\hline gobierno & 4.345 & 4.010 & 3.711 & 3.413 & 2.332 & 1.802 & 1.175 & 1.824 & 350 & 22.962 \\
\hline quiero & & 3.477 & 3.249 & 3.593 & 2.995 & 3.088 & 1.884 & 2.339 & 656 & 21.281 \\
\hline chadwickrenuncia & & 2.403 & 4.354 & 3.929 & 3.747 & 2.204 & 1.810 & 2.132 & 304 & 20.883 \\
\hline canal & 2.654 & 4.193 & 4.003 & 2.747 & 1.471 & 1.454 & 962 & 1.292 & 406 & 19.182 \\
\hline salir & 2.075 & 2.785 & 2.307 & 3.116 & 2.405 & 2.663 & 1.556 & 1.974 & & 18.881 \\
\hline chileviolateshumanrights & & & & & 9.818 & 5.101 & 1.045 & 1.741 & 466 & 18.171 \\
\hline mierda & 2912 & 2856 & 24.96 & 2.994 & 2128 & 1701 & 1119 & 1386 & 410 & 18002 \\
\hline
\end{tabular}

\section{Discusión}

En este apartado describiremos dos áreas que serán analizadas desde el punto de vista empírico, por un lado, las investigaciones relacionadas con los movimientos sociales de las dos últimas décadas en Chile, y por otro, la teoría del discurso político y del significante vacío. En el primero, se encontró una gran cantidad de literatura que describe el surgimiento, en las dos últimas décadas, de una diversidad de demandas sociales, desde los movimientos secundarios del año 2006 en adelante. La segunda área que mencionamos tiene relación con la propuesta teórica de Ernesto Laclau y Chantal Mouffe, los cuales serán útiles para comprender el rol de las palabras en el contexto de fuertes protestas sociales.

La primera reflexión parte con contexto nacional de las dos últimas décadas, en Chile han ocurrido una serie de estallidos sociales ligados al movimiento 
estudiantil (Agacino, 2013; Aguilera, 2011, 2014; Avendaño, 2014; Mayol y Azócar, 2011), al movimiento feminista (Zerán, 2018), al movimiento ecologista (Allain, 2019), al movimiento indígena (Marimán, 2012) y al movimiento de trabajadores (Boccardo y Ruiz, 2009, 2012; Garcés, 2012). Estos acontecimientos han marcado tres décadas completas, desde que finalizó la Dictadura Militar de Augusto Pinochet (Fleet, 2011; Thielemann, 2011), sin embargo, han sido las últimas dos las que han presentando mayor impacto. Estas demandas siguieron estando latentes, porque la mayoría no están resultadas, son demandas que no han sido atendidas por el sistema político (Jiménez-Yañez, 2020). Las demandas frustradas se han ido acumulando en el tiempo, provocando que previo al estallido existiera un malestar latente (Mayol, 2019; Akram, 202O; Ruiz, 202O; Alé, Duarte y Miranda, 2021).

En este sentido, una serie de temáticas sociales se han expresado en los distintos movimientos sociales (Garretón, 2002), desde el sistema educativo hasta las leyes de más igualdad para las mujeres (Sotomayor, 2018). En base a esto, podemos señalar que la primera característica que identificamos es la existencia de una corriente muy amplia de sectores que han mostrado un malestar social o disconformidad con el sistema político-económico, lo cual no se expresa en los indicadores de participación en las elecciones en las dos últimas décadas (PNUD, 2014), muy por el contrario, la participación de la sociedad en política ha través de las manifestaciones callejeras y métodos no convencionales ha presentado un aumento y una continuidad en el tiempo (Villalobos, 2021). Además, este amplio malestar reúne una importante cantidad de jóvenes de sectores acomodados, más ligados a las demandas ecológicas y feministas (Gayo y Méndez, 2021), por ende, hablamos de una serie de movimientos heterogéneos en demandas y con un carácter pluriclasista. Sin embargo, una parte del motor del malestar se encuentra en las condiciones del modelo económico (Akram, 2020), por ejemplo, las cifras entregadas por el Programa de las Naciones Unidas para el Desarrollo (2017) dan cuenta de importantes brechas económicas y sociales en el país, las cuales generan guetos e identidades muy marcadas en los territorios y comunidades.

Este escenario de conflictos fue anticipado por el sociólogo Tomás Moulian (2002), al analizar los problemas que se desarrollarían a partir de la constitución impuesta por la Dictadura cívico-militar, la cual crearía una sociedad en la medida del crédito para el consumo, una dimensión que sería criticada en las movilizaciones del año 2011 (Mayol, 2012). A esto se suma un sistema que funciona con bajos salarios, una alta desigualdad de clase, de raza y de género, y con una ciudadanía complicada por sus deudas (Ruiz y Boccardo, 2015; Ffrench-Davis, 2014). Este contexto permite acercarnos de forma más clara a lo conceptos y problemáticas que están presentes en los posts de Twitter que se capturaron para esta investigación.

En síntesis, las transformaciones neoliberales implementadas por la Dictadura cívico-militar se perpetuaron en la constitución del 80, lo cual 
funcionó como un dique de contención de las demandas sociales, especialmente por el alto quorum para cambiarlas (Tomás Moulian, 2002). Por este motivo, la aparición de los movimientos sociales y la generación de una serie de demandas que se fueron posicionando cada vez con más fuerza en las dos últimas décadas (Mayol, 2019; Akram, 2020; Ruiz, 2020; Alé, Duarte y Miranda, 2021). Una diversidad de demandas chocó con el muro constitucional y la estructura política que resguarda al sistema económico, por eso fueron frustradas de forma constante, lo cual impidió una canalización de las demandas sociales por parte de la institución y la transformación de las condiciones de vida de la ciudadanía.

La segunda área abordada tiene relación con la manifestación del malestar en el campo de la comunicación y el discurso durante el estallido social. Es importante comenzar describiendo las características de las protestas sociales anteriores y la particularidad del fenómeno ocurrido el 18 de octubre. En este sentido, las distintas expresiones de los movimientos sociales en Chile presentan peculiaridades dependiendo del momento, no solamente por los actores, sino también por el tipo de herramientas comunicacionales que se han empleado para dar conocer las demandas de la organización. Por ejemplo, en las últimas tres décadas se pueden observar los estallidos de $1997^{3}, 2002^{4}, 2006^{5}, 2011^{6}, 2018^{7}$ y $2019^{8}$; todos estos momentos tenían algún canal que facilitaba la información y la difusión de las demandas, sin embargo, las herramientas más recientes de telefonía móvil con acceso a internet han sido las más transformadoras (Subirats, 2015). Entre los años 1997 y 2002 la comunicación instantánea era nula por parte de los jóvenes; mientras que en el año 2006 surge el formato de blog como forma de información, especialmente el Fotolog; luego, en el 2011, los estudiantes de las universidades y colegios utilizaron el FaceBook, el YouTube y el Twitter, estos se transformaron en los nuevos espacios públicos; y en el 2018 y 2019 se sumaron las plataformas de Instagram y Whatsapp, sin embargo, estas plataformas ya habían sido utilizadas en movilizaciones de menor envergadura entre el 2012 y el 2017. Sin embargo, esta investigación está limitada al estudio de la red social Twitter, dado que nos interesa recuperar las palabras claves que estuvieron presentes en la primera semana del estallido.

Antes de ahondar en el tema, debemos señalar las características del movimiento, puesto que tiene en su mayoría son expresiones inorgánicas (sin un típico liderazgo de partido o de otra forma de organización), a diferencia de los movimientos anteriores del año 2006 y 2011, los cuales tenían una relación directa con los nuevos partidos políticos y los partidos tradicionales (Mella, Ríos y Rivera, 2016). En paralelo a este fenómeno se observa el uso de nuevas tecnologías, como los blogs que se usaron para organizar y difundir las demandas

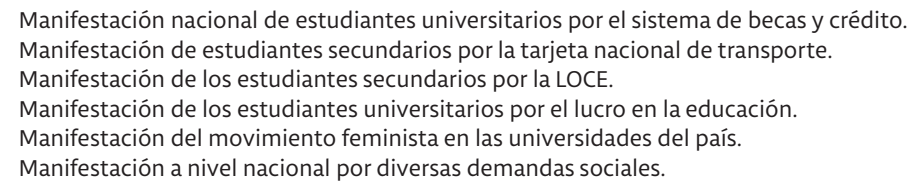


del movimiento, este fue el caso del movimiento estudiantil secundario. Esta idea la desarrolla Manuel Castell (2000), en sus tres volúmenes de "La era de la información", en sus libros, explica como la tecnología ha permitido crear una comunidad más conectada y en red, permitiendo una forma de protesta amplificada, o sea nuevas formas de expresar la democracia.

Las nuevas tecnologías han permitido mejor la comunicación. En la actualidad las redes sociales son herramientas que los ciudadanos utilizan de forma diaria. Durante la primera semana del estallido social, que va desde el 18 al 26 de octubre, las manifestaciones no se expresaron solamente en las calles, sino que también en las redes sociales. En Chile los datos entregados por La Encuesta Nacional Bicentenario Universidad Católica - GfK Adimark (2016), señalaba que el uso de celular en Chile llegó al 92\%, mientras que el acceso a internet, según los datos de la Subtel (2017), estaban en 87,5\%. Por otro lado, los el $42 \%$ de los usuarios de internet señaló que su principal fuente de información son las redes sociales (Bicentenario Universidad Católica - GfK Adimark;2016). A pesar de estos datos, al observar el nivel de uso y conocimiento de Twitter es una de redes sociales menos presentes, ya que es una plataforma que prioriza el debate, esto queda claro con la figura 1.

Figura 2. Redes sociales en Chile

\begin{tabular}{|c|c|c|c|c|c|c|c|c|c|c|}
\hline \multirow[b]{2}{*}{ Red Social } & \multicolumn{5}{|c|}{ Conoce } & \multicolumn{5}{|c|}{ Usa } \\
\hline & $\begin{array}{c}\text { TOTAL } \\
2017\end{array}$ & 16 a 29 & 30 a 44 & 45 a 59 & $\begin{array}{l}600 \\
\text { más }\end{array}$ & $\begin{array}{l}\text { TOTAL } \\
2017\end{array}$ & 16 a 29 & 30 a 44 & 45 a 59 & $\begin{array}{l}60 \text { o } \\
\text { más }\end{array}$ \\
\hline WhatsApp & $88,2 \%$ & $97,9 \%$ & $96,1 \%$ & $87,3 \%$ & $65,2 \%$ & $79,4 \%$ & $91,1 \%$ & $88,5 \%$ & $75,4 \%$ & $47,1 \%$ \\
\hline Facebook & $87,4 \%$ & $97,8 \%$ & $96,5 \%$ & $84,9 \%$ & $64,0 \%$ & $65,5 \%$ & $82,0 \%$ & $77,0 \%$ & $55,6 \%$ & $29,2 \%$ \\
\hline YouTube & $76,8 \%$ & $93,0 \%$ & $87,9 \%$ & $71,1 \%$ & $46,4 \%$ & $43,3 \%$ & $55,8 \%$ & $46,6 \%$ & $36,3 \%$ & $24,9 \%$ \\
\hline Google & $75,3 \%$ & $90,8 \%$ & $86,2 \%$ & $70,2 \%$ & $45,4 \%$ & $48,7 \%$ & $58,2 \%$ & $51,5 \%$ & $42,5 \%$ & $35,6 \%$ \\
\hline Twitter & $59,2 \%$ & $79,5 \%$ & $70,2 \%$ & $46,3 \%$ & $32,3 \%$ & $10,7 \%$ & $15,9 \%$ & $12,5 \%$ & $7,0 \%$ & $3,8 \%$ \\
\hline Instagram & $58,0 \%$ & $83,6 \%$ & $70,1 \%$ & $44,2 \%$ & $23,4 \%$ & $21,9 \%$ & $44,4 \%$ & $19,0 \%$ & $10,2 \%$ & $3,6 \%$ \\
\hline Snapchat & $27,9 \%$ & $54,8 \%$ & $32,3 \%$ & $12,6 \%$ & $4,2 \%$ & $3,2 \%$ & $6,0 \%$ & $3,5 \%$ & $1,4 \%$ & $0,2 \%$ \\
\hline Linkedln & $21,7 \%$ & $32,6 \%$ & $30,4 \%$ & $11,8 \%$ & $7,2 \%$ & $3,7 \%$ & $3,9 \%$ & $6,0 \%$ & $2,2 \%$ & $1,6 \%$ \\
\hline Pinterest & $20,3 \%$ & $33,6 \%$ & $26,6 \%$ & $9,5 \%$ & $6,9 \%$ & $3,6 \%$ & $4,9 \%$ & $4,4 \%$ & $1,7 \%$ & $2,4 \%$ \\
\hline Otra & $0,2 \%$ & $0,2 \%$ & $0,1 \%$ & $0,4 \%$ & $0,1 \%$ & $0,1 \%$ & $0,2 \%$ & $0,1 \%$ & $0,0 \%$ & $0,1 \%$ \\
\hline Ninguna & $8,0 \%$ & $0,2 \%$ & $1,7 \%$ & $8,2 \%$ & $27,0 \%$ & $9,5 \%$ & $0,5 \%$ & $2,3 \%$ & $11,5 \%$ & $36,5 \%$ \\
\hline
\end{tabular}

Fuente: Elaboración Subtel (2017)

Twitter a diferencia de las otras redes tiene un componente muy atractivo para los líderes de opinión política en Chile, ya que es el espacio en que la mayoría de las personas más activas o interesadas en política genera contenidos. Las principales temáticas del momento se posicionan con una palabra o frase que tenga una frecuencia alta y que sea novedosa para el algoritmo de 
Twitter. Por esta razón, es una plataforma muy usada por los políticos, ya que pueden visibilizar sus temáticas a través de la posibilidad de ser tendencia. Además, esta herramienta fue clave para entregar información al instante de las manifestaciones que estaban ocurriendo en todo el país (Jiménez-Yañez, 2020). Por este motivo, la investigación utilizará la red social Twitter, ya que es la herramienta que mejor captura el debate político y la inmediatez de los acontecimientos.

El análisis del contexto nacional y contenido que se captura en Twitter permite comprender el problema propuesto en este trabajo, el discurso y el significado de las palabras. Esta investigación, toma como referencia los estudios de Ernesto Laclau y Chantal Mouffe (2005), principalmente la idea de "significante vacío", antagonismo y momento populista. El primer término es claves, ya que expresa y constituye una cadena de equivalencia de demandas. El momento de equivalencia genera lazos integradores entre las demandas, pues, si bien hay equivalencia tan sólo porque existe una pluralidad de demandas, el momento de equivalencia no está meramente subordinado a ellas, sino que juega un rol crucial en hacer posible esa pluralidad. En este sentido, el "significante vacío" sería el símbolo que permitiría reunir las demandas del pueblo, esto provocaría la conformación de un antagonista, cristalizando un momento populista que representa la disputa simbólica del sentido común de las personas.

\section{Resultados}

En este apartado, se presentarán los resultados de mayor relevancia respecto de los análisis descriptivos, relacionados con la hipótesis planteada. Contando con la base de datos optimizada y estructurada, se procesa la información de acuerdo a los criterios que se definieron en la investigación; en la figura 3 se muestra la relevancia de las palabras recopiladas durante todo el período, en formato de nube de palabras, en las cuales ya se puede identificar de manera visual los términos que describen el fenómeno asociado. En la nube de palabras se pude observar que la figura del presidente Piñera toma mayor relevancia, por ende, el malestar es canalizado hacia la figura del presidente. Sin embargo, también se puede apreciar que existe una diversidad de referencias al presidente Sebastián Piñera, por ejemplo, el \#renunciapiñera. 
Figura 3. Nube de Palabras

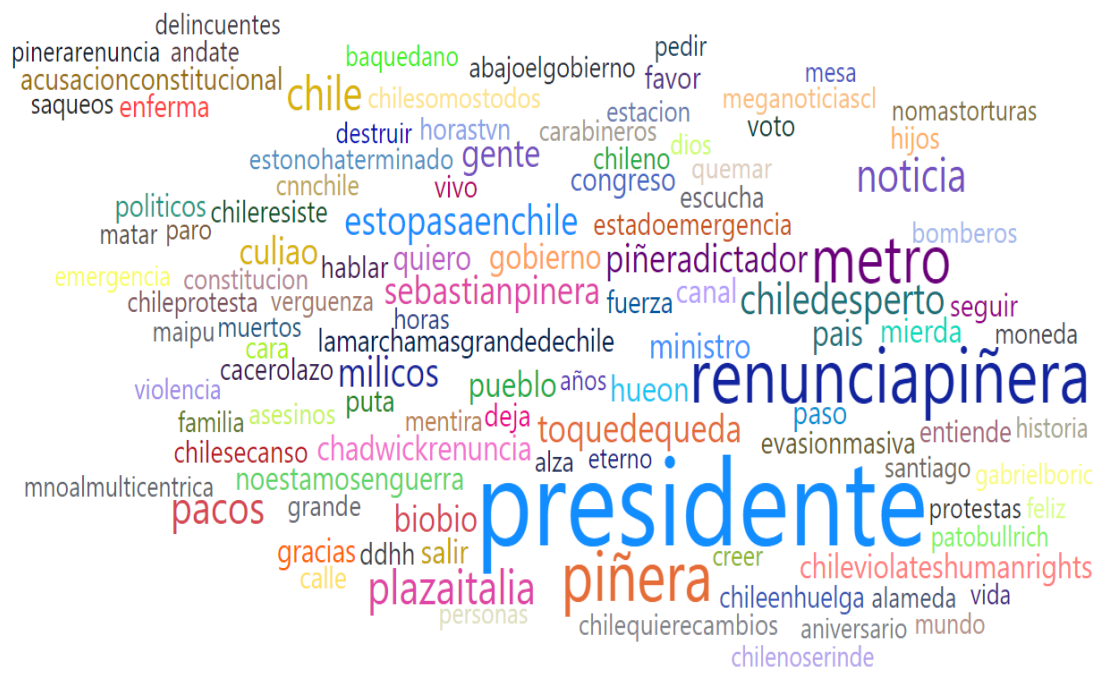

Fuente: Elaboración propia

Con el fin de cuantificar el impacto relativo entre los términos encontrados, se elaboró el histograma mostrado en la figura 4, donde ya aparece el término "presidente" como el protagonista del estudio. Pero también vemos como otros significantes vacíos se comienzan a expresar en el histograma, especialmente para la configuración de la idea pueblo en términos de Ernesto Laclau.

Un ejemplo son los siguientes hashtags: \#chiledesperto, \#PlazaItalia, \#ChileDespertó y \#Pueblo. Entonces. Las palabras nos permiten identificar en estos "significantes vacíos" el proceso de unión de las demandas mencionadas en la investigación y a la vez encontrar al "significante vació" que cumple el rol de antagonista de las distintas demandas de la ciudadanía. 
Figura 4. Histograma de Frecuencias

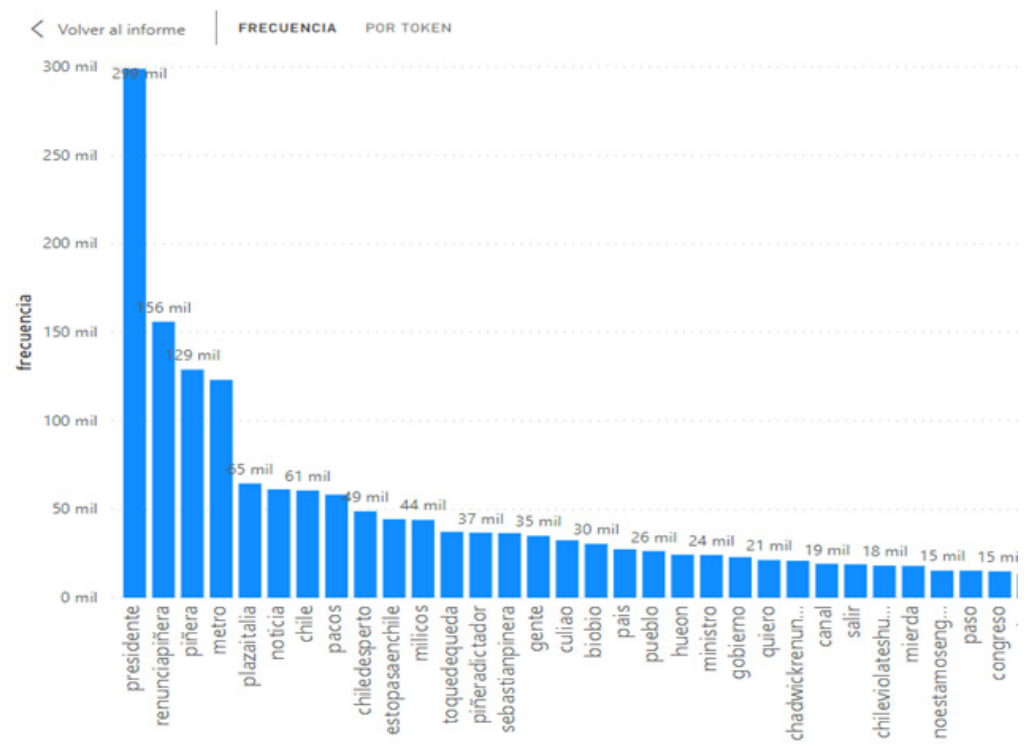

Fuente: Elaboración propia

Otra forma de visualizar este efecto consiste en el análisis del formato llamado Treemap, donde muestra la distribución relativa de los términos encontrados, dimensionados por el tamaño de los cuadros de influencia que se encuentran en la figura 5 , y donde se aprecia de forma clara la gran variedad de palabras encontradas y el efecto de las 10 primeras, que ya representan sobre el $50 \%$ del universo de frecuencias. Esto es muy relevante porque podemos observar como se configuraron los significantes durante los primeros días de la protesta, algunas palabras como Plaza Italia o Chile Despertó, tendrán un peso particular, ya que serán íconos de la protesta social en los siguientes meses. 
Figura 5. Diagrama de Treemap

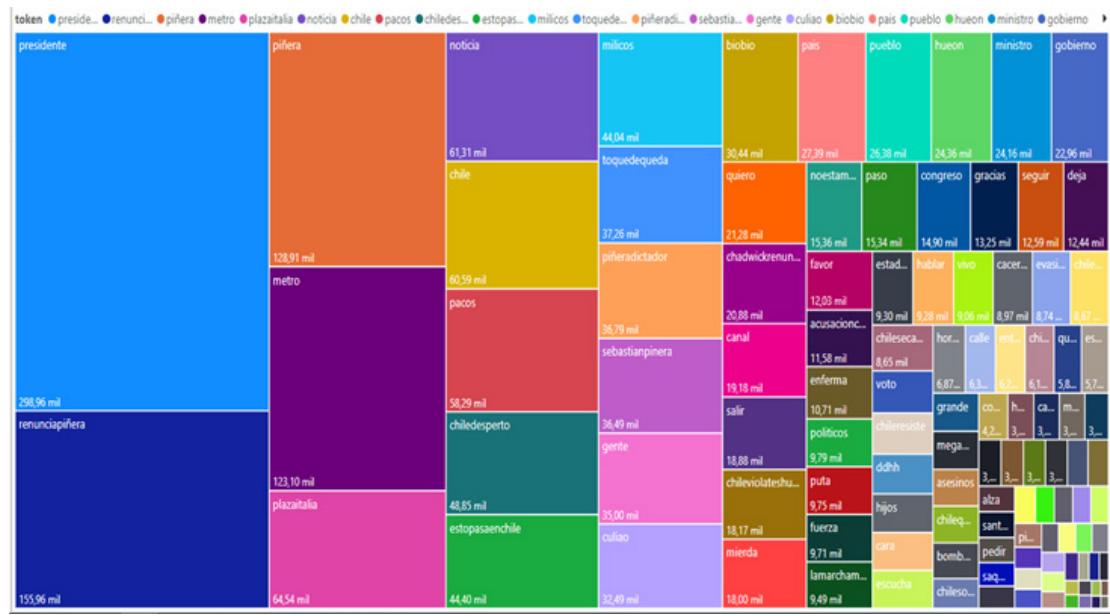

Fuente: Elaboración propia

Teniendo una idea clara del fenómeno a nivel global, en la figura 6 se introduce una nueva variable temporal al estudio, que permite describir la evolución de las reacciones durante los días analizados del conflicto:

Figura 6 Histograma de Frecuencia Temporal

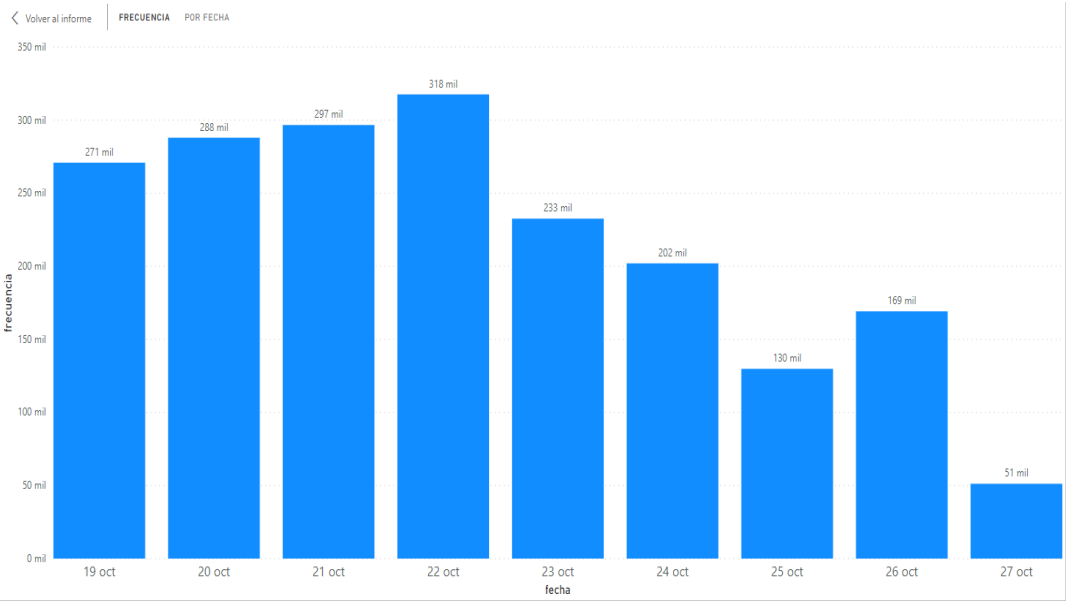

Fuente: Elaboración propia

Por último, se generó un diagrama en la figura 7 con un efecto de las frecuencias acumuladas de palabras y su evolución en el tiempo para evaluar la tendencia y el peso relativo de cada término en el escenario global analizado: 
Figura 7. Diagrama Temporal de Frecuencia Acumulada

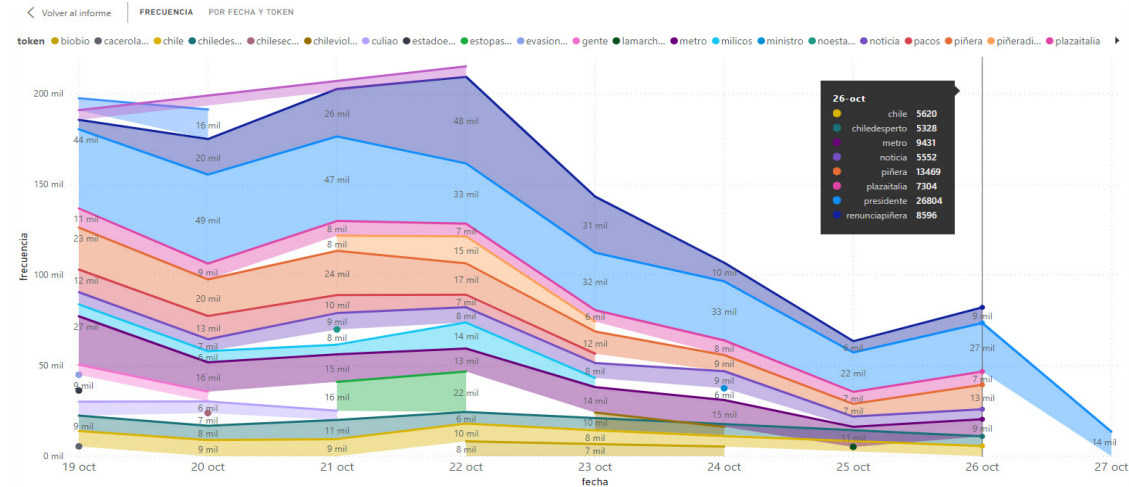

Fuente: Elaboración propia

Basado en el análisis anterior, se presentan algunos resultados aplicando filtros que permitan describir la evolución de emociones relativas al fenómeno asociado, dentro de las cuales se presentan 3 categorías que se destacan por su efecto patente en los hechos acontecidos. La figura 8 representa el resultado que se relaciona con los sentimientos de violencia, donde se destaca la expresión "noestamosenguerra", producto de la declaración nacional de este estado en el discurso presidencial.

Figura 8. Evolución de términos relacionados con el sentimiento de "violencia"

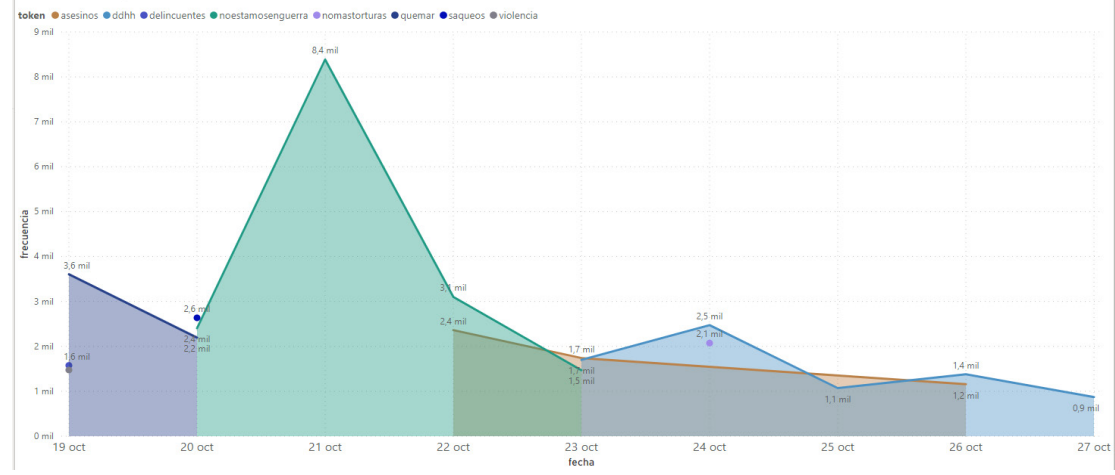

Fuente: Elaboración propia

La figura 9 muestra el resultado del análisis considerando valores positivos y de apoyo, destacándose de forma evidente el concepto "Chile" como símbolo de la unidad en los tiempos de crisis: 
Figura 9. Evolución de términos relacionados con sentimientos positivos y de apoyo

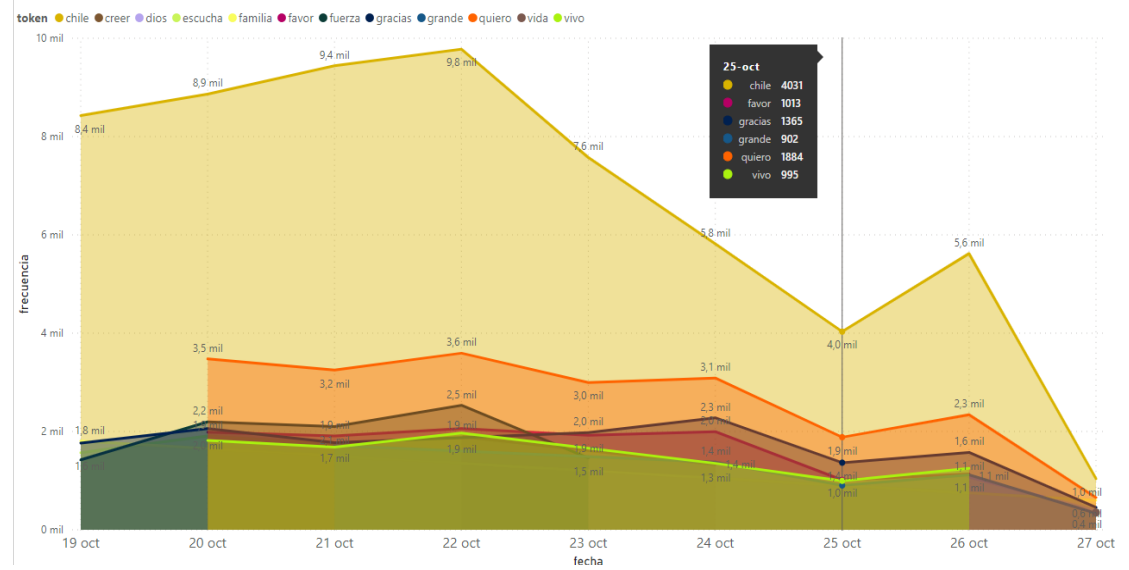

Fuente: Elaboración propia

Por otro lado, el rol de las instituciones y los personajes más relevantes que influyen en el descontento social, se muestra en la figura 10, como indicador de factor de cambio en la sociedad, destacándose de forma estable las palabras "piñera" y "chadwick", seguidos de las fuerzas de orden y el metro como medio de transporte

Figura 10. Evolución de términos relacionados con sentimientos relativos a personajes e instituciones

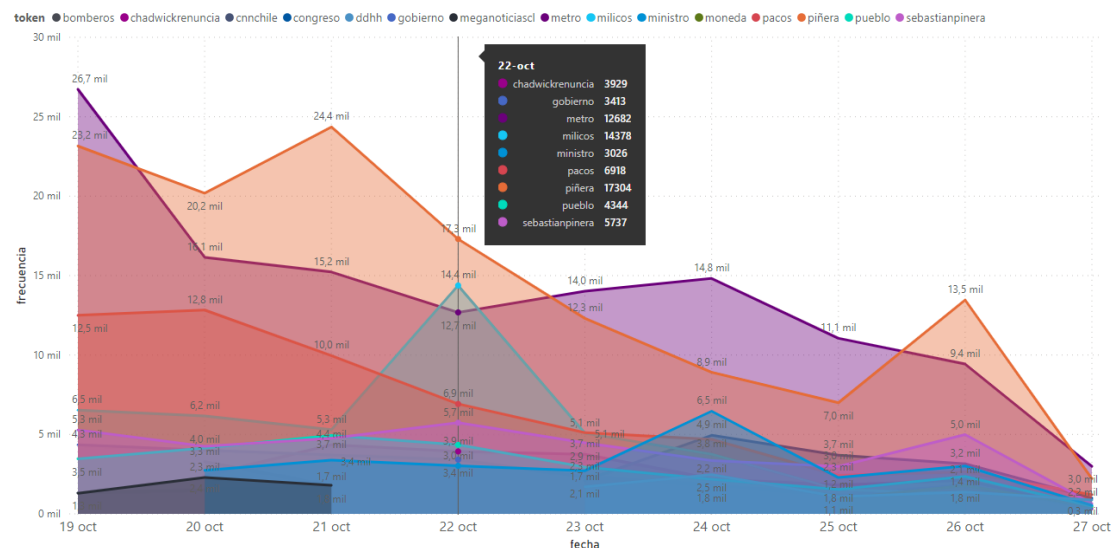

Fuente: Elaboración propia 


\section{Conclusiones}

Las conclusiones que podemos desarrollar van en la línea de lo que presentan los datos de forma muy evidente. Lo primero tiene relación con los conceptos claves que están en los gráficos. La figura del presidente es la más mencionada en los posts de Twitter, esto nos indica que gran parte del malestar se canaliza en él, por ende, cumple el rol de antagonista. En segundo lugar, podemos identificar que existieron más significantes vacíos, los cuales en un primer momento hegemonizaron la discusión, chile despertó y plaza Italia -que después se renombrará como Plaza Dignidad- tendrán un rol muy importante en la canalización de la idea pueblo en términos de populismo; de igual forma, carabineros, el ministro Andrés Chadwick y las fuerzas armadas tendrán un significado particular, ya que fueron identificados con la reprehensión por parte del Estado. En tercer lugar, esto permite identificar un momento impugnador a la elite y al modelo político-económico de Chile, esta impugnación es un momento populista, en el cual los "significantes vacíos" dotan de contenido a las figuras públicas, las instituciones y los espacios físicos. Por lo cual, esto quiere decir que existe una relación entre la propuesta de Ernesto Laclau y Chantal Mouffe, y el fenómeno del estallido social ocurrido en Chile. Por este motivo, la investigación realizada es muy útil, ya que se proceso un gran volumen de opiniones y referencias al contexto de la primera semana de las protestas. La minería de opinión resultó ser una herramienta útil para capturar y procesar la información. En un próximo estudio buscaremos pasar a una fase más detallada de text mining y de interpretación de emociones, ya que este primer artículo de investigación busca explorar en las palabras claves que se generaron durante la primera semana de movilización.

\section{Referencias bibliográficas}

Agacino, R. (2013). “Movilizaciones estudiantiles en Chile. Anticipando el futuro". Educação em Revista, Marília, Vol. 14, $\mathrm{n}^{\circ} 1$, pp. 7-20.

Aguilera, O. (2011). "Movidas, movilizaciones y movimientos de juventudes". En Latinoamericamente. Santiago: Editorial Quimantú, pp. 119-129.

Aguilera, O. (2012). "Repertorios y ciclos de movilización juvenil en Chile (2000-2012)". Utopía y Praxis: Revista internacional de filosofía iberoamericana y teoría social, $\mathrm{n}^{\circ} 57$, pp. 101 - 108.

Akram, H. El Estallido. Santiago de Chile: El Desconcierto.

Alé, S., Duarte, K. y Miranda, D. (2021). Saltar el torniquete: Reflexiones desde las juventudes de octubre. Santiago de Chile: Fondo de Cultura Económica Chile.

Allain, M. (2019). Conflictos y protestas socio-ambientales en Chile: Reflexiones metodológicas y resultados. Revista de Sociología, Vol. 34, nº 1, pp. 81-101.

Avendaño, O. (2014). "Fracturas y representación política en el movimiento estudiantil: Chile 2011”. Última década, vol 22, nº 41, pp. 41-68. 
Bennett, W. (2003), "Communicating Global Activism". Information, Communication and Society, $\mathrm{n}^{\circ}$ 6, pp. 143-168.

Bicentenario Universidad Católica - GfK Adimark (2016). Encuesta Nacional Bicentenario. Centro de Políticas Públicas UC y del Instituto de Sociología de la Pontificia Universidad Católica de Chile, en conjunto con GfK Adimark.

Boccardo, G y Ruiz, C. (2009). "Problemas sociales de la concentración económica (visto desde la crisis)". Revista Análisis. Departamento de Sociología de la Universidad de Chile, $\mathrm{n}^{0} 12$, pp. 31-54.

Boccardo, G y Ruiz, C. (2012). "Peripecias del capital y el trabajo en el neoliberalismo avanzado". Revista Análisis. Departamento de Sociología de la Universidad de Chile, $\mathrm{n}^{\circ} 15$, pp. 25-52.

Bourdieu, P. (1985). ¿Qué significa hablar? Economía de los intercambios lingüísticos. Madrid, AKAL.

Bourdieu, P. (1999). Intelectuales, política y poder. Buenos Aires, EUDEBA.

Castells, M. (2000). La era de la información: Economía sociedad y cultura. El poder de la identidad Vol. II. Madrid: Alianza Editorial.

Claret, A. (2015). "Asociaciones, movimientos y redes, El continuum de la participación juvenil 2015”. En Subirats, J. Ya nada será lo mismo, Los efectos del cambio tecnológico en la política, los partidos y el activismo juvenil (p. 28-44). Madrid-España: Centro Reina Sofía sobre Adolescencia y Juventud.

Ffrench-Davis, R. (2014). Chile entre el neoliberalismo y el crecimiento con equidad: cuarenta años de políticas económicas y sus lecciones para el futuro. Santiago, Chile: JC Sáez Editor SpA.

Fleet, N. (2011). Movimiento estudiantil y transformaciones sociales en Chile: una perspectiva sociológica. Revsita Polis, $\mathrm{n}^{\circ} 30$, pp. 2-13.

Gayo, M. y Méndez, M. (2021). La movilización de los jóvenes "cuicos" entendida desde los procesos socialización. En S. Alé, S., K. Duarte y D. Miranda, Saltar el torniquete: Reflexiones desde las juventudes de octubre (pp. 124-129). Santiago de Chile: Fondo de Cultura Económica Chile.

Garcés, M. (2012). El despertar de la sociedad. Santiago de Chile: LOM ediciones.

Garretón, M. (2002). La transformación de la acción colectiva en América Latina. Revista de la CEPAL. $n^{\circ}$ 76, pp. 7-23.

Jiménez-Yañez, C. (2020). “\#Chiledespertó:causas del estallido social en Chile”. Revista Mexicana de Sociología 82, núm. 4 (octubre-diciembre, 2020): 949-957.

Kuhn, T. (1962). La estructura de las revoluciones científicas. Madrid: Fondo de Cultura Económica.

Lakatos, I. (1983). La metodología de los programas de investigación científica. Madrid: Alianza Editorial.

Laclau, E. (1978). Política e ideología en la teoría marxista: Capitalismo, fascismo, populismo. España: Siglo XXI Editores.

Laclau, E. (1985). Hegemonía y estrategia socialista: Hacia una radicalización de la democracia. Argentina: Fondo de Cultura Económica de Argentina.

Laclau, E. (2005). La razón populista. Argentina: Fondo de Cultura Económica de Argentina. Laclau, E. (2008). Debates y combates. Argentina: Fondo de Cultura Económica de Argentina. Le Bon, G. (1985). the crowd: a study of the popular mind. South Carolina, US: Createspace Mayol, A. (2012). El derrumbe del modelo. Santiago: LOM ediciones. 
Mayol, A. y Azócar, C. (2011). Politización del malestar, movilización social y transformación ideológica: el caso "Chile 2011". Polis, nº30, pp. 1-19.

Mayol, A. (2019). Big Bang, Estallido social 2019. Santiago: Catalonia.

Marimán, J. (2012). Autodeterminación: Ideas políticas mapuche en el albor del siglo XXI. Chile: LOM.

Mendoza, M., Poblete, B. y Castillo, C. (2010). "Twitter Under Crisis: Can we trust what we rt?". Workshop on Social Media Analytics, v. (1), pp. 71-79.

Mella, M. Ríos, H., Rivera, R., (2016). Condiciones orgánicas y correlaciones de fuerza del movimiento estudiantil chileno. Una aproximación desde la Confech (2011-2015). Revista Izquierdas, $\mathrm{n}^{\circ} 27$, pp. 124-16o.

Miranda, C. y Castillo, J. (2021). Cambios en la participación política de los escolares. En S. Alé, S., K. Duarte y D. Miranda, Saltar el torniquete: Reflexiones desde las juventudes de octubre (pp. 51-60). Santiago de Chile: Fondo de Cultura Económica Chile.

Moulian, T. (2002). Chile Actual. Anatomía de un mito. LOM; Santiago.

Programa de Naciones Unidas para el Desarrollo, (2014). Auditoría a la Democracia. Chile: PNUD.

Programa de Naciones Unidas para el Desarrollo, (2017). Desiguales: Orígenes, cambios y desafíos de la brecha social en Chile. Santiago de Chile: Programa de las Naciones Unidas para el Desarrollo.

Ruiz, C. y Boccardo, G. (2015). Los chilenos bajo el neoliberalismo: clases y conflicto social. Santiago, Chile: Ediciones y Publicaciones El Buen Aire.

Sotomayor, F. (2018). "Malestar, acción colectiva y movimientos sociales en chile: (2001 2017)". ReonFacema, Vol. 4, nº 1, pp. 1137-1151.

Thielemann, L. (2011). "Para una periodificación del movimiento estudiantil de la transición (1987- 2011)”. En: Sistematización de Talleres para la Acción Estudiantil. CEFECH-Heinrich-Böll- Stiftung. Santiago.

Tilly, Ch. y Wood, L. (2010). Los movimientos sociales: 1768-2008. Desde sus orígenes a Facebook. Barcelona: Crítica.

Touraine, A. (1993). La Voix et le regard. Sociologie des movements sociaux. París: Seuil.

Touraine, A. (2006). "Los movimientos sociales". Revista colombiana de sociología, n⿳0027, pp. 255-278.

Subtel (2017). IX Encuesta Accesos y Usos de Internet. Chile: Subtel.

Villalobos, C. (2021). Una continuidad discontinua: análisis retrospectivo del 18-O a la luz del ciclo de protestas juveniles en el campo educativo. En S. Alé, S., K. Duarte y D. Miranda, Saltar el torniquete: Reflexiones desde las juventudes de octubre (pp. 41-47). Santiago de Chile: Fondo de Cultura Económica Chile.

Vinck, D. (2018). Humanidades Digitales: La cultura frente a las nuevas tecnologías. Barcelona: Gedisa.

Zerán, F. (2018). Mayo feminista: La rebelión contra el patriarcado. Chile: LOM. 